\title{
“Te explicó qué quiere decir”, “te digo cómo se llama”. Interacciones niño-niño en torno a vocabulario no familiar
}

\author{
Florencia Alam, Dra. Alejandra Stein, Dra. Celia R. Rosemberg \\ Universidad de Buenos Aires y Consejo Nacional de Investigaciones Científicas y \\ Técnicas, Buenos Aires, Argentina
}

(Artículo recibido el 12 de julio de 2011; versión final recibida el 27 de octubre de 2011)

\begin{abstract}
Este trabajo se propone analizar los movimientos conversacionales a los que recurren niños/as de 12 años para explicar vocabulario a niños/as de 4 años en situaciones de alfabetización. Las situaciones fueron video filmadas en escuelas en la que se llevaba a cabo un programa de niños tutores. Los niños/as participantes del programa vivían en poblaciones urbano-marginadas en Buenos Aires, Argentina. El corpus de datos analizados incluye 50 situaciones de tutoría. La unidad de análisis estuvo constituida por intercambios en los que un niño/a mayor explicaba una o más palabras, denominaba o intentaba elicitar un término determinado por parte del pequeño/a. Para el análisis de los datos se empleó un procedimiento cualitativo (Glaser y Strauss, 1967; Strauss y Corbin, 1991). Los resultados mostraron que en estas situaciones los niños/as mayores recurren no solo a información lingüística sino también a información de otros campos semióticos.
\end{abstract}

\section{Introducción}

En el presente trabajo ${ }^{1}$ se analizan situaciones de intercambio entre niños/as, que tuvieron lugar en el marco de un programa de niños tutores en alfabetización con el objeto de identificar y de describir los movimientos interaccionales por medio de los cuales los niños/as tutores denominan o explican el significado de palabras poco familiares a los niños/as pequeños/as. El trabajo se enmarca en una perspectiva que asume la interrelación entre la cognición, el lenguaje y el contexto social (Vygotsky, 1964, 1978; Bruner, 1986; Rogoff, 1993; Nelson, 1996, 2007). Esta perspectiva resulta particularmente pertinente para el estudio del aprendizaje del vocabulario, en tanto diversas teorías psicolingüísticas actuales coinciden en señalar que el aprendizaje de vocabulario constituye un proceso sociocultural (Nelson, 1996, 2007; Tomasello, 1998; Weizman y Snow, 2001), un proceso que se inicia a partir de los esfuerzos del niño/a por descubrir el intento referencial de las otras personas en situaciones de interacción social. 
En el marco de este encuadre teórico las investigaciones han resaltado la importancia que adquieren los intercambios conversacionales para el aprendizaje de vocabulario; tanto en relación a la diversidad y la complejidad del vocabulario que se presenta a los niños/as como en relación al sostén que este contexto proporciona para el aprendizaje. La mayoría de estas investigaciones, se han centrado en los intercambios niño/a - adulto (Beals y Tabors, 1995; Weizman y Snow, 2001, entre otros) y han puesto de manifiesto cómo los adultos contribuyen a crear contextos en los que los niños/as pueden más fácilmente inferir e interpretar el significado de las palabras que desconocen.

Algunos trabajos sobre la interacción adulto - niño/a realizados en el medio familiar (Rosemberg y Stein, 2009; Rosemberg, Stein y Borzone, 2011) y otros realizados en el medio escolar (Danis, Bernard y Leproux, 2000; Torr y Scot, 2006; Rosemberg, 2007; Rosemberg y Silva, 2009) muestran que las situaciones compartidas de lectura de cuentos constituyen actividades en las que se incrementa la calidad de las propiedades lexicales del entorno lingüístico al que se hallan expuestos los niños/as y en las que los adultos ponen en juego una variedad de movimientos interaccionales para explicar a los niños/as palabras poco familiares.

Por su parte, los trabajos que analizan el impacto de la interacción entre pares en el desarrollo y el aprendizaje (Forman y Cazden, 1984; Azmitia, 1988; Verba, 1998; Chi y Roy, 2010, entre otros) se focalizan, en general, en la forma de colaboración que se produce durante situaciones de resolución de problemas; pero se centran generalmente en los resultados y no en la negociación de los procesos de colaboración en la tarea. Los trabajos toman como objeto de estudio tanto tareas cognitivas desarrolladas en interacciones cooperativas por niños/as de igual edad (Annis, 1983; Tudge, 1992; Topping, 2005, entre otros) como los procesos de tutoría que se producen cuando sujetos de diferente edad o experticia interactúan para resolver conjuntamente una tarea (Juel, 1996; Kermani y Moallem, 1997; Topping y Bryce, 2004, entre otros).

Las metodologías planteadas para el estudio de los procesos de tutoría implican tanto la implementación de un programa en el que los tutores reciben entrenamiento sobre cómo llevar a cabo la tutoría (King, Staffieri y Adelgais, 1998), como en contextos naturales (Roscoe y Chi, 2004). Los resultados en su mayoría muestran un 
progreso tanto en los aprendices como en los tutores (Schrader y Valus, 1990; Berliner y Casanova, 1998).

Aún cuando en muchas de estas investigaciones las tareas conllevan intercambios verbales entre los niños/as participantes para arribar a la resolución, no se analizan los recursos lingüísticos y de otros campos semióticos (Goodwin, 1980, 2000) a los que los niños/as recurren en la interacción con el objeto de alcanzar la comprensión mutua (Gumperz, 1982, 1984). El objetivo de este trabajo es precisamente analizar, en situaciones de alfabetización generadas a partir de lecturas de cuentos, los intercambios entre el tutor (un niño/a mayor) y el aprendiz (un niño/a pequeño/a) en los que para arribar a la comprensión mutua se negocia el significado de las palabras, a la vez que se generan contextos de oportunidades para el aprendizaje lingüístico y discursivo.

\section{Metodologia}

Corpus

El corpus de datos analizados incluye 50 situaciones de tutoría niño/a - niño/a realizadas en el marco del programa “De niño a niño: un programa de niños tutores en alfabetización” (Rosemberg y Alam, 2009 - en curso). En las situaciones participaron niños y niñas de 4 años (aprendices) y niños y niñas de 12 años (tutores) provenientes de poblaciones urbano-marginadas en Buenos Aires, Argentina.

En las situaciones de tutoría, los tutores y los aprendices realizaban de manera conjunta distinto tipo de actividades de alfabetización, así como actividades focalizadas en el desarrollo de los conocimientos y habilidades implicados en el sistema de escritura.

El programa de niños tutores en alfabetización tiene por objeto capitalizar las interacciones entre niños/as de diferentes edades para conformar una matriz que andamie el inicio del proceso de alfabetización de los más pequeños y genere un contexto de lectura y escritura significativo en el que los niños/as mayores desarrollen un mayor dominio del sistema de escritura, mejorando sus habilidades como lectores y escritores.

La implementación del programa se llevó a cabo en escuelas a las que asistían niños/as y adolescentes pertenecientes a contextos urbano-marginados. En talleres 
semanales los coordinadores pedagógicos y miembros del equipo de investigación capacitaban a los niños/as mayores para llevar a cabo la tutoría: se leían los cuentos que luego leerían a los pequeños, se pensaban y discutían estrategias destinadas a mantener la atención del pequeño/a y se los estimulaba a formular distinto tipo de preguntas. Asimismo, se reflexionaba en torno al vocabulario en los textos y acerca de cómo explicar el significado de palabras desconocidas a un niño/a pequeño. En una segunda instancia semanal se realizaba la actividad de tutoría: cada uno de los niños/as más grandes leía a uno de los pequeños/as de jardín de infantes los cuentos contenidos en uno de los libros de la serie “En la Casa de Oscarcito” (Rosemberg y Borzone, 2008) y realizaba las actividades y los juegos propuestos en el libro.

\section{Obtención y transcripción de la información empírica}

Las situaciones fueron audio y video filmadas en escuelas en la que se llevaba a cabo el programa de niños tutores. Las grabaciones fueron transcritas para su análisis, realizando una transcripción literal de los archivos de audio. Los registros fueron complementados con la información del contexto situacional y comportamientos no verbales registrados en los videos. Las transcripciones fueron realizadas siguiendo las convenciones descritas en el Anexo 1.

\section{Procedimientos de análisis de los datos}

La unidad de análisis estuvo constituida por intercambios en los que un niño mayor explicaba una o más palabras, denominaba o intentaba elicitar un término determinado por parte del pequeño.

Para el análisis de los datos se empleó un procedimiento cualitativo, el Método Comparativo Constante (Glaser y Strauss, 1967; Strauss y Corbin, 1991). Dicho procedimiento permitió la elaboración inductiva de categorías o tipos de movimientos conversacionales que ponen en juego en la interacción los niños/as de 12 años (tutores) para explicar vocabulario a los niños/as de 4 años (aprendices) en situaciones de alfabetización.

El análisis que dio lugar a la elaboración de las categorías fue realizado conjuntamente por las tres investigadoras, a partir del estudio del $20 \%$ de las situaciones. Cada una de ellas codificó luego independientemente el 30\% de los casos. 
En un 93,3\% de los casos acordaron en los códigos asignados. Luego de discutir los criterios para categorizar los casos en los que no se había llegado a un acuerdo en la codificación, el 50\% restante de las situaciones fue codificado por la primera autora.

\section{Resultados y Discusión}

Los resultados mostraron que en las situaciones en las que los niños/as mayores se enfrentan con la necesidad de explicar a los pequeños/as vocabulario poco frecuente recurren, en la generación de un marco de atención conjunta para la elaboración del significado, no sólo a información lingüística sino también a gestos así como también a información de las ilustraciones en el libro o de los objetos en la situación. Por otra parte, el análisis puso de manifiesto que los aprendices también recurren no sólo al lenguaje verbal sino también a los gestos y la proxemia para participar en la situación de alfabetización y mostrar atención e involucramiento en la tarea.

Así por ejemplo, en el siguiente intercambio Javier, el tutor, recurre a diferentes tipos de información para elicitar un término por parte de Ezequiel. A su vez, Ezequiel, el aprendiz, se vale de múltiples recursos para mostrar su participación en la actividad.

\section{Extracto (1)}

Situación: Javier (12) y Ezequiel (4) resuelven juntos una actividad en la que deben completar las partes que le faltan al dibujo de una gallina.

1. Javier: ¿qué partes le faltan a la gallina? (0.1) ¿qué es esto? ((señala la cara de la gallina)) (0.2) ¿qué le falta acá? ((señala la pata que le falta a la gallina))

2. Ezequiel: em:::: ((acerca más la cara al libro y mira con atención))

3. Javier: ¿cómo se llama esto? ((señala la pata que le falta a la gallina))

4. Ezequiel: ((mira lo que Javier señala))

5. Javier: ¿esto qué es? ((señala el pie del niño))

6. Ezequiel: ((mira su pie, lo mira a Javier, vuelve a mirar a la gallina y señala la parte que falta)) el PIE (.) le falta ((Javier ayuda a Ezequiel a escribir la palabra $))^{2}$

7. Javier: ((vuelve a la página donde está la gallina incompleta y señala con el lápiz el ala que falta)) ¿qué le falta? ¿le falta el ala?

8. Ezequiel: ((señala el dibujo))

9. Javier: mirá ésta ((señala la gallina completa)) y mirá ésta ((señala la gallina incompleta)) ¿qué es esto? ((señala el ala))

10. Ezequiel: ((se acerca más al libro)) e:::: 
11. Javier: ¿cómo se llama con la que vuela?

12. Ezequiel: alas

En el fragmento presentado Javier (el tutor) y Ezequiel (el niño pequeño) deben completar las partes del dibujo de una gallina. Javier comienza preguntándole a Ezequiel por una de esas partes: la pata -¿qué partes le faltan a la gallina? (0.1) ¿qué es esto? ((señala la cara de la gallina)) (0.2) ¿qué le falta acá? ((señala la pata que le falta a la gallina))- (turno 1). Como el niño no sabe la respuesta, el tutor produce una autorreparación (Schegloff, Jefferson y Sacks, 1977) y en su nueva pregunta desplaza el foco de lo que el objeto es, a su denominación (turno 3) -¿cómo se llama esto?-. De este modo, intenta restablecer la comunicación entre ambos; necesaria para lograr la consecución de la actividad. Sin embargo, Ezequiel continúa sin saber la respuesta. Por lo tanto, el tutor recurre estratégicamente a su propio cuerpo (turno 5) - ¿esto qué es? ((señala el pie del niño))- y, en este caso, el niño pequeño puede responder (turno 6). Tal como se muestra en el análisis Ezequiel responde recurriendo a información proveniente de una multiplicidad de campos: visualmente, en tanto que mira primero a su interlocutor y luego el objeto nombrado; gestualmente a través del señalamiento hacia la parte aludida; y verbalmente a través de la respuesta verbal -el PIE le falta-. Se pone de manifiesto de este modo la cooperación y el involucramiento del niño pequeño en la tarea de alfabetización que están llevando a cabo.

Más adelante en el intercambio para elicitar el término ala, Javier explícitamente solicita al niño que compare la información en ambas ilustraciones: la que presenta la gallina incompleta cuyas partes deben escribir y la que presenta la gallina completa (turno 9) -mirá ésta y mirá ésta-. Sin embargo, Ezequiel no sabe la respuesta, por lo que el tutor produce nuevamente una autorreparación de su pregunta inicial. En este caso, formula una pregunta cerrada en la que proporciona la función del objeto para solicitar la denominación (turno 11) -¿cómo se llama con la que vuela?-. Nuevamente el movimiento de reparación que realiza Javier, que en esta ocasión se centra en información lingüística, permite que el niño pequeño resuelva la actividad.

Por su parte, Ezequiel, el niño pequeño, a pesar de no responder verbalmente a las preguntas anteriores de Javier (turnos 7 y 9) -¿qué le falta?; ¿le falta el ala?; ¿qué es esto?- sí lo hace de forma proxémica, al acercar su cuerpo al libro y mirar mostrando 
atención hacia lo señalado por el tutor (turno 10), y de forma gestual, al señalar las imágenes (turno 8). Estas intervenciones no verbales ponen de manifiesto y comunican al tutor su involucramiento en la actividad de alfabetización. Cabe señalar, en este sentido, que un análisis que atienda sólo a los recursos verbales de los participantes no podría dar cuenta, en este caso, de la participación del niño pequeño (Goodwin, 1980).

Como se observa en el intercambio presentado el tutor recurre en su andamiaje a lo que Goodwin (2007) define como gestos asociados con el entorno; esto es, a gestos que no pueden entenderse sin tener en cuenta el contexto en el que están inmersos. En efecto, para poder entender las preguntas de Javier es necesario tener en cuenta las imágenes indicadas por el gesto, pero a su vez, Javier recurre al señalamiento y al uso de deícticos como una forma de resolver la tarea que implica su rol de tutor: ayudar al niño pequeño a completar la actividad, ya que el gesto y las formas deícticas le permiten guiar la producción del pequeño sin nombrar el objeto.

La referencia al cuerpo de los niños, que se ha mostrado en el ejemplo anterior, es un recurso del que los tutores se valen con frecuencia. En algunos casos los niños mayores utilizan este recurso creando de forma explícita un paralelismo entre el cuerpo de los pequeños y el vocabulario expuesto en el texto. Así por ejemplo, en el siguiente intercambio Mariano (12) explica el término “forzudo” a través de una comparación con el propio cuerpo de Ariel (4).

\section{Extracto (2)}

Situación: Mariano (12) le lee un cuento a Ariel (4) sobre un zapallo gigante.

1. Mariano: ((lee)) y dicho esto salió a buscar su camioneta con la ayuda de cinco vecinos muy for-forzudos (.) ¿sabés lo que es forzudos?

2. Ariel: ¿qué?

3. Mariano: lo que- viste que vos tenés acá fuerza ((señala el brazo de Ariel)) vos podés levantar esto ((muestra el lápiz que tiene en la mano)) ellos tenían mucha fuerza y ellos pudieron levantar la- el gran zapallo

En el intercambio Mariano se focaliza en el vocabulario por medio de una pregunta “¿sabes lo que es forzudos?” (turno 1). Dado que Ariel no sabe la respuesta (turno 2) Mariano explica el término yuxtaponiendo información que proporciona el contexto -el propio cuerpo del niño-, información verbal (turno 3) -viste que vos tenés acá fuerza- y 
gestual -((señala el brazo de Ariel))-. Asimismo, Mariano complejiza la explicación del significado de la palabra proporcionando información sobre las propiedades funcionales del concepto: especifica aquello que la fuerza le permite realizar -vos podés levantar esto-. Cabe señalar que esta información resulta importante para la tarea que están conjuntamente realizando, en la medida en que le permite al niño comprender la relación, planteada en el cuento, entre los vecinos forzudos y el zapallo gigante. Al final de su emisión, Mariano retoma la situación del cuento (turno 3) -ellos tenían mucha fuerza y ellos pudieron levantar la- el gran zapallo-, con el anclaje que proporciona la explicación previa que formuló haciendo referencia al cuerpo del niño. De este modo, los movimientos conversacionales del tutor generan un contexto en el que el niño puede tanto comprender el cuento a la vez que se configuran oportunidades para que el niño aprenda nuevas palabras.

En otras ocasiones los tutores introducen palabras nuevas que no estaban presentes en el texto. En estos intercambios los tutores suelen proporcionar una explicación para el nuevo término retomando, en la mayoría de los casos, la situación planteada en el texto, tal como se observa en el siguiente intercambio en el que Matías (12) introduce un término nuevo: atajo.

\section{Extracto (3)}

Situación: Matías (12) le lee el cuento de Caperucita Roja a Dylan (4).

1. Matías: ((lee)) el lobo hambriento pensó en comerse a Caperucita allí mismo pero tuvo miedo porque cerca había unos leñadores cortando leña entonces el lobo pensó en un plan para poder comerse a Caperucita le-le preguntó donde quedaba la casa de su abuelita y decidió tomar el camino más corto para llegar antes que Caperucita ((detiene la lectura y mira a Dylan)) (.) ¿sabés lo que es tomar un atajo?

2. Dylan: ((asiente))

3. Matías: ¿qué es?

4. Dylan: e:::

5. Matías: cuando Caperucita va por un camino más largo y el lobo va por un camino más corto ((marca con el dedo en la hoja el camino que está dibujado))

6. Dylan: ((señala la hoja del libro, apoya el dedo sobre la hoja y lo mueve formando una línea recta)) 
En el intercambio presentado, el tutor, Matías, detiene la lectura del cuento e introduce, de modo explícito por medio de una pregunta un término menos familiar que el presentado en el cuento (turno 1) - ¿sabés lo que es tomar un atajo?-. Como Dylan no conoce el término (turno 4), el niño mayor proporciona una explicación que retoma la situación planteada en el cuento (turno 5) -cuando Caperucita va por un camino más largo y el lobo va por un camino más corto-. Cabe señalar que esta explicación conlleva por parte del tutor la explicitación de una condición que está implícita en el relato: para que el lobo tome el camino más corto Caperucita tiene que ir por el camino más largo. A su vez, el tutor, de forma implícita, yuxtaponiendo de modo adyacente (Lemke, 1997) la locución incluida en el texto -tomar un camino- y otra que constituye en este caso particular un sinónimo -va por un camino- que resulta más familiar.

Cabe destacar que luego de la respuesta de Matías, Dylan señala la imagen del cuento y con el dedo sobre la hoja forma una línea recta (turno 6), reproduciendo el gesto producido por Matías en su explicación del término “atajo” (turno 5). El hecho de que el niño retome el gesto producido por el tutor podría ser entendido en la situación comunicativa como una confirmación de que ha comprendido la explicación que le ha proporcionado el tutor.

\section{Conclusión}

El análisis de las situaciones de tutoría en alfabetización realizado en el marco de este trabajo mostró que en los intercambios entre el tutor y el aprendiz la referencia a las imágenes de los textos, el cuerpo de los niños, los objetos presentes, así como el uso de gestos se yuxtaponen a la información lingüística. A este respecto cabe sostener como Duranti (1997) que resulta necesario concebir la interacción como una arquitectura en red, en tanto que comunicamos secuencial y/o simultáneamente a través de diferentes recursos: gestos, movimientos corporales, habla, entonación, la interacción con el entorno material y el espacio físico. Tal como se pone de manifiesto en las situaciones analizadas, con el sostén que proporcionan estos intercambios los niños/as pueden tener mayores oportunidades de atender a las palabras menos familiares que se presentan en los cuentos y de comprender su significado.

La importancia que tienen las situaciones de tutoría en términos de las oportunidades que ellas generan para el desarrollo de un vocabulario diverso y complejo 
debe ser ponderada, como sostienen Rosemberg y Stein (2009), en el marco de las investigaciones que han comprobado que el vocabulario facilita el acceso y el dominio de la lectura y de la escritura en la escuela primaria (Biemiller, 2006; Perfetti, 2007; Protopapas, Sideridis, Mouzaki y Simos, 2007) así como también el carácter predictivo que posee el aprendizaje temprano de vocabulario sobre las habilidades de escritura que muestran en niños y niñas de poblaciones urbano-marginadas (Rosemberg, Stein y Menti, en prensa).

Por otra parte, cabe señalar, también que estas situaciones de tutoría, en tanto conducen a los tutores a reflexionar acerca de los términos que explican, pueden constituir también un contexto en el que aprendan a partir de la explicación que llevan a cabo y del auto monitoreo que realizan en la situación (Roscoe y Chi, 2004). Se constituyen, de este modo, en situaciones significativas en las que los niños/as mayores pueden ampliar su vocabulario y, consecuentemente, mejorar sus habilidades para producir una prosa expandida oral o escrita.

\section{Referencia Bibliográficas}

Annis, L. (1983). The processes and effects of peer tutoring. Human Learning, 2, 3947.

Azmitia, M. (1988). Peer interaction and problem solving: when are two heads better than one? Child Development, 59, 87-96.

Beals, D.B., \& Tabors, P.O. (1995). Arboretum, bureaucratic and carbohydrates; Preschoolers' exposure to rare vocabulary at home. First language, 18, 57-76.

Berliner, D., \& Casanova, U. (1998). Peer tutoring: A new look at a popular practice. Instructor, 97 (5), 14-15.

Biemiller, A. (2006). Vocabulary development and instruction: A prerequisite for schooling learning. En D.K. Dickinson \& S.B. Neuman (Eds.), Handbook of Early Literacy Research, Vol. 2 (pp. 41-51). New York: The Guilford Press.

Bruner, J. (1986). El habla del niño. Barcelona: Paidos.

Chi, M.T.H., \& Roy, M. (2010). How adaptive is an expert human tutor? En J. Kay, \& V. Aleven (Eds.), International Conference on Intelligent Tutoring Systems (ITS '10), (pp. 401-412).

Danis, A., Bernard, J.M., \& Leproux, C. (2000). Shared picture-book reading: A sequential analysis of adult - child verbal interactions. British Journal of Developmental Psychology, 18, 369-388.

Duranti, A. (1997). Linguistic Antropology. Cambridge: Cambridge University Press.

Forman, E., \& Cazden, C. (1984). Perspectivas vygotskianas en la educación: el valor cognitivo de la interacción entre iguales. Infancia y Aprendizaje, 27-28, 139157. 
Glaser, B., \& Strauss, A. (1967). The discovery of grounded theory. Chicago: Aldine Publishing Company.

Goodwin, C. (1980). Restarts, pauses, and the achievement of a state of mutual gaze at turn-beginning. Sociological Inquiry, 50 (3-4), 272-302.

Goodwin, C. (2000). Action and embodiment within situated human interaction. Journal of Pragmatics, 32, 1489-1522.

Goodwin, C. (2007). Participation, stance and affect in the organization of activities. En Discourse \& Society. London: Sage Publications. Vol 18 (1) 53-73.

Gumperz, J. (1982). Discourse strategies. Cambridge: Cambridge University Press.

Gumperz, J. (1984). Commucative competence revisted. En D. Schiffrin (Ed.) Meaning, form and use in context: Lingüistics applications. Washington: Georgetown University Press.

Juel, C. (1996). Learning to learn from effective tutors. En L. Schauble \& R. Glaser (Eds.), Innovations in learning: New environments for education (pp. 49-74). Mahwah, NJ: Erlbaum.

Kermani, H., \& Moallem, M. (1997). Cross-age tutoring: Exploring features and Processes of Peer mediated learning. Dialogue From the Field, 4 (3), 453-485.

King, A., Staffieri, A., \& Adelgais, A. (1998). Mutual peer tutoring: effects structuring interaction to scaffold peer learning. Journal of Educational Psychology, 90 (1), $134-152$

Lemke, J. (1997). Aprender a hablar ciencia. Barcelona: Paidós.

Nelson, K. (1996). Language in cognitive development. Cambridge: Cambridge University Press.

Nelson, K. (2007). Young minds in social worlds. Experience, meaning and memory. Cambridge, MA: Harvard University Press.

Perfetti, C. (2007). Reading ability: Lexical quality to comprehension. Scientific Studies of Reading, 11(4), 357-383.

Protopapas, A., Sideridis, G.D., Mouzaki, A., \& Simos, P.G. (2007). Development of lexical mediation in the relation between reading comprehension and word reading skills in Greek. Scientific Studies of Reading, 11(3), 165-197.

Rogoff, B. (1993). Aprendices del pensamiento. Barcelona: Paidós.

Roscoe, R.D., \& Chi, M. (2004). The influence of the tutee in learning by peer tutoring. En K. Forbus, D. Gentner \& T. Regier (Eds.), Proceedings the 26th Annual Meeting of the Cognitive Science Society. London: Psychology Press, pp. 11791184.

Rosemberg, C.R. (2007). Nuevas palabras en situaciones de lectura de cuentos en la escuela infantil. Ponencia presentada en el XXXI Congreso Interamericano de Psicología - Por la integración de las Américas. Ciudad de México, México, 1 a 5 de julio de 2007.

Rosemberg, C.R. \& Borzone, A.M. (2008). Serie de libros infantiles "En la Casa de Oscarcito". Fundación Care (Alemania), Fundación Arcor (Argentina).

Rosemberg, C.R., \& Silva, M.L. (2009). Teacher - children interaction and concept development in kindergarten. Discourse processes, 46(6), 572-591.

Rosemberg, C.R., \& Stein, A. (2009). Vocabulario y alfabetización temprana. Un estudio del entorno lingüístico en hogares de poblaciones urbano-marginadas. En M.C. Richaud \& J.E. Moreno (Eds). Investigación en Ciencias del Comportamiento, pp. 517-541, Buenos Aires: Ediciones CIIPME-CONICET. 
Rosemberg, C. R.; Stein, A. \& Borzone, A. M. (2011). Lexical input to young children from extremely poor communities in Argentina. Effects of a home literacy program. Journal of Early Childhood Research, 9 (1), 36-52.

Rosemberg, C.R. \& Alam, F. (2009-en curso). "De niño a niño: un programa de niños tutores en alfabetización." Programa de intervención en escuelas y centros comunitarios. Financiado por: Fundación Care (Alemania) - Fundación Save the Children (Argentina) - CONICET (Argentina) - SECyT (Argentina).

Rosemberg, C.R., Stein, A., \& Menti, A. (en prensa). Orientación educativa sobre el vocabulario y el acceso a la alfabetización. Evaluación de impacto de un programa de intervención en las familias y en la escuela. Revista Internacional e Interdisciplinaria de Orientación Vocacional Ocupacional.

Schegloff, A., Jefferson, G., \& Sacks, H.E. (1977). The preference for self-correction in the organization of repair in conversation. Language, 53 (2), 361-382.

Schrader, B., \& Valus, A. (1990). Disabled learners as able teachers: a cross-age tutoring project. Academic Therapy, 25, 589-597.

Strauss, A., \& Corbin, J. (1991). Basics of cualitative research. Grounded theory. Procedures and technics. London: Sage Publications.

Tomasello, M. (Ed.). (1998). The new psychology of language: Cognitive and functional approaches to language structure. Mahwah, NJ: Lawrence Erlbaum Associates.

Topping, K.J. (2005). Trends in peer learning. Educational Psychology, 25 (6): 631645.

Topping, K., \& Bryce, A. (2004). Cross-age peer tutoring of reading and thinking: Influence on thinking skills. Educational Psychology, 24 (5), 595-621.

Torr, J., \& Scott, C. (2006). Learning 'special words': Technical vocabulary in the talk of adults and preschoolers during shared reading. Journal of Early Childhood Research, 4 (2), 153-167.

Tudge, J.R.H. (1992). Processes and consequences of peer collaboration: A Vygotskian analysis. Child development, 63, 1364-1379.

Verba, M. (1998). Tutoring between young children: How symmetry can modify asymmetrical interactions. International Journal of Behavioural Development, 22 (1), 195-216.

Vygotsky, L.S. (1964). Pensamiento y lenguaje. Buenos Aires: Lautaro.

Vygotsky, L.S. (1978). Mind in society. Cambridge, MA: Harvard University Press.

Weizman, Z.O., \& Snow, C. (2001). Lexical input as related to children's vocabulary acquisition: Effects of sophisticated exposure and support for meaning. Developmental Psychology, 17, 265-279.

\section{Anexo 1: Glosario de símbolos de transcripción utilizados}

(( )) fenómenos no léxicos

(.) pausa breve, menor a 0.1 segundos

(0.1) pausa prolongada: los números entre paréntesis indican el tiempo de la pausa en segundos

:: alargamiento de sonido vocálico o consonántico

PALABRA EN MAYÚSCULAS énfasis

- interrupción

( ) palabra ininteligible

$\dot{¿}$ ? entonación interrogativa 


\section{Referencias de las autoras}

Florencia Alam es licenciada en Letras por la Universidad de Buenos Aires. Actualmente cursa la Maestría en Análisis del Discurso y el Doctorado en Educación, ambos en la Universidad de Buenos Aires, con una beca doctoral del Consejo Nacional de Investigaciones Científicas y Técnicas en Argentina (CONICET).

\section{Email: florenciaalam@gmail.com}

Alejandra Stein es doctora en Ciencias del Lenguaje por la Universidad de Córdoba. Actualmente realiza su post doctorado con una beca del Consejo Nacional de Investigaciones Científicas y Técnicas en Argentina (CONICET).

Celia Renata Rosemberg es investigadora independiente del Consejo Nacional de Investigaciones Científicas y Técnicas en Argentina (CONICET) y Profesora Adjunta concursada en la Universidad de Buenos Aires.

$1 \quad$ Un estudio en el marco de un Programa de Niños Tutores en Alfabetización en poblaciones urbanomarginadas en Argentina.

2 No transcribimos el fragmento en el que el tutor ayuda a escribir la palabra porque no resulta pertinente a los fines del presente trabajo. 